EPJdirect A1, 1-11 (2018)

DOI $10.1007 / \mathrm{s} 1010518 \mathrm{a} 123$

EPJdirect

electronic only

(C) Springer-Verlag 2018

\title{
LHC Symposium 2003: Summary Talk
}

\author{
J. A. Appel \\ Fermilab, PO Box 500, Batavia, IL 60510, USA \\ e-mail: appel@fnal.gov \\ Received:
}

\begin{abstract}
This summary talk reviews the LHC 2003 Symposium, focusing on expectations as we prepare to leap over the current energy frontier into new territory. We may learn from what happened in the two most recent examples of leaping into new energy territory. Quite different scenarios appeared in those two cases. In addition, we review the status of the machine and experiments as reported at the Symposium. Finally, I suggest an attitude which may be most appropriate as we look forward to the opportunities anticipated for the first data from the LHC.
\end{abstract}

PACS: 12.15.-y;13.85.-t;12.60.-i;14.80.Bn

As we contemplate the three days of excellent talks we have just experienced, we are invited to think about how to convey our science and its goals to the public. In that context, we should understand where the public perceptions are. I am reminded of a recent discussion among knowledgeable people, motivated by the book "The End of Science" by John Horgan. In it, Horgan says "And now that science - true, pure, empirical science - has ended, what else is there to believe in?" It is too bad that anyone thinking this was not here at this Symposium! We are here reaffirming that empirical science is alive and well.

\section{Leaping Over Energy Frontiers}

Even more than simply continuing the empirical research of the past, we are at the threshold of a new era, with a new leap beyond the current energy frontier. Following the excellent presentations at this Symposium, it is perhaps worthwhile to pause a moment and consider our most recent leaps of energy frontiers. What do they suggest?

What happened when the ISR and the " $200 \mathrm{GeV}$ " machine turned on? Available center-of-mass energy jumped from $8 \mathrm{GeV}$ to $20-50 \mathrm{GeV}$. New energy territory opened to us. We were surprised, even shocked by how different the world seemed. Almost immediately, we saw the advent of high- $p_{t}$ events at both the CERN ISR and at Fermilab. Pions were observed with cross sections no longer dropping exponentially with $p_{t}$. 11, 2] Rather, the drop with $p_{t}$ was more like a power-law, eventually reaching that for hard point-like scattering! Backgrounds for many planned experiments were orders of magnitude larger than expected. More fundamentally, we observed (as we now understand it) the effects of the quark substructure of hadrons. 
We also started to produce particles essentially undreamed of before - well, dreamed of by only a few foolhardy visionaries. In addition to the pions at high $p_{t}$ coming from hadronic interactions, a plethora of leptons appeared. Their numbers could not be explained by the decay of known strongly-produced particles. 3 Eventually, these leptons were seen to come from the semileptonic decays of the previously-unknown heavy quarks.

Perhaps the excess of leptons reminds you of the apparent excesses of heavy quarks seen in hadronic interactions today (especially of B mesons and $J / \psi$ and $\psi^{\prime}$ onia). It may be that what Mary Bishai referred to as a $b$-production excess of 1.2-1.9 times theory, [4] will continue to fall as theoretical models of production are refined. The fractional excess does seem to be coming down with time. However, it is possible that we are already seeing the effects of something which we will only understand once we have data from the LHC.

What happened when the big CERN and Fermilab hadron colliders turned on? Available energy jumped from a few tens of $\mathrm{GeV}$ to 630 and 2,000 GeV. Again, new energy territory opened for exploraton. We were again surprised - maybe not so much by a new energy scale which was predicted ( $W$ and $Z$ masses), but by the very large mass of the top quark. I remember well, how upon seeing evidence for the bottom quark, we immediately expected to see the top quark at $\pi$ times the mass of the bottom quark, just like the factor between the bottom quark and the strange quark. The ratio of top to bottom quark masses is more like 40 than 3 ! We do not understand why the top quark is so heavy to this very day.

We have seen no direct evidence of any of the suggested new particles: not sequential $W$ or $Z$ bosons, not Higgs, not SUSY, nor techni-particles. We have not seen a break in $p_{t}$ spectra, nor the onset of a new level in the hierarchy of matter, nor any suggestion of something more fundamental than quarks and leptons.

\section{How the Preparations are Going}

As you have shown at this symposium, you are building detectors, and solving technical and managerial problems. You are also building expanded collaborations and new tools to deal with the new sociology: learning how to live with larger and increasingly internationalized collaborations, learning new techniques and tools for ever larger projects, and beginning to experiment with new computing paradigms like GRID. [5] 6]

I have been impressed by the trigger tables shown and the expanding physics goals shown by experiments. We had talks on heavy-ion collision measurements in the big $p-p$ detectors, ATLAS and CMS, detection of quark jets in ALICE, and the appearance of B physics everywhere. Detectors have had design and engineering updates, and simulations continue to include more complete detector modeling. As usual, the results suggest somewhat less capability, but hopefully more realistic expectations. At the same time, perhaps motivated in part by the new understanding, better algorithms have been developed to compensate for somewhat reduced detector expectations; e.g., in tracking and and heavy quark tagging algorithms. In order to continue this progress, mock data challenge efforts 
cannot be over-valued, both for improving the physics reach of experiments and and for debugging the computing environment of the future. Even more, better motivation will come from the data itself once you have the real thing.

As an example of how time with actual physics data helps, let me cite the work reported by Juan Estrada at a seminar at Fermilab just the week before the Symposium, and referred to by Jianming Qian. [7] Unlike previous CDF and DZero top-quark mass analyses that used templates, this new DZero analysis uses lepton plus jets events and makes a direct calculation of the signal and background probability for each event. That probability depends on all measured momenta of the final state lepton and jets, and each event's contribution depends on how well it is measured. The quoted preliminary result for the top quark mass is $M_{t}=180.1 \pm 3.6 \pm 4.0 \mathrm{GeV}$. The improvement in statistical error is equivalent to a factor of 2.4 in the size of the data sample. The relative error in this one decay channel alone is $3 \%$, compared to $2.9 \%$ from the previous combined CDF and DZero average for all analysed decay channels.

\section{Progress, Yet Concerns}

You have shown real, substantial progress from the past year at this Symposium. It has been very good to see the progress on the LHC itself, and on the detectors, software, and physics planning. We can all be happy that civil construction is now going well, and magnet production is getting better. Roger Cashmore spoke of the "nightmare" of the civil construction problems that are now behind us. We are also happy to see so many detector components getting into construction. We have heard about facing real challenges. Some have been technical; e.g., in military radiation-hard electronics, some electronic noise and yield issues, material budgets, and radiation damage effects. Some challenges have been financial in origin, leading to scope changes and, sometimes, to additional funding. Other challenges have been with schedules, requiring continuous review and adjustments (e.g., lack of test-beam availability). Personally, I am happy to see some full system tests, and indications that planning for commissioning is getting serious attention. We are all happy to see solutions over the last year to these and other problems .

Your progress is important to us at Fermilab. First, it is important for our physics program (CMS) and our super-conducting magnet program. Mike Witherell, in his Director's welcome to you, noted that only Fermilab's Tevatron Collider and neutrino programs are larger here at the Lab. Second, your progress is important for the planning of much of the rest of our program as well. For the Tevatron Collider, currently the energy frontier machine, the importance is obvious. However, in fact, your progress is important to all of HEP. Consider the implications for B Factories!

Nevertheless, even as an LHC outsider, I have concerns. The scale of the industrial technology needed for the machine and for the detectors is still new to our community. It is not obvious that accelerator components will stay ahead of the "just in time" schedule. We have all been invited to look at the CERN LHC "dashboard" on the web. [8] There is no real evidence yet of the rapid change in delivery slopes needed to meet the schedule for beams. 
Timing can be everything. Staging of detector components may get us to the point where we cannot buy needed components later. This is already a problem. Some commercial technologies may not last long enough for our development and construction schedules. DMILL radiation hard ASIC technology is going away already; will 0.25 micron ASIC technology be far behind? Even networking and computing components are a worry. Consider the "Objectivity" software suite. At the same time, there are more technology decisions yet to be made than is healthy at this stage. I would mention the CMS pixel size, the ATLAS B-layer pixel size, the CMS electromagnetic calorimeter electronics, and the LHCb RICH photon-detection decision especially.

Common computing approaches can save duplication, and help by stressing systems in more than one environment. This can head off problems later, as the environments evolve for all those using a given approach. Yet, this commonality of approach is just starting. I was surprised in the talks of Lothar Bauerdick [5] and Nick Brook $[\underline{6}$ to see so far how little integrated into the big experiments are the GRID projects.

Testing and commissioning times are getting squeezed almost everywhere already!

\section{A Few Words About the Physics}

The main message from the experiments at this Symposium has been that discoveries may be made very early, if nature is as expected by most. In my summary, I have chosen to show some of the most frequently referenced transparencies. Since these plots have been shown so often, I won't even have to tell you what they are, even if none of us remembers or cites where the plots were first shown! The famous Higgs sensitivity plot is made to show the early discovery expected over the whole range of likely mass. Recently, including the vector-boson fusion process helps in the previously-difficult low-mass region. For SUSY, again we are assured of rapid discovery, the plots showing possible discovery up to $1.5 \mathrm{TeV}$ even with only " 1 day" of data - OK, it's a good day.

In the area of Heavy Ions, I'd like to mention the particularly good review of Heavy Ion Physics at RHIC by Gunther Roland. 9] He showed a "consistent description of the final state," but noted that "we're missing a picture of [the] dynamical evolution" that gets us there from the initial conditions. Many speakers showed where the LHC sits on the phase diagram of temperature vs baryonic chemical potential. This plot does not do justice in my eyes to the role of the LHC. The LHC is shown in a tiny corner of the plot. Yet, the "missing picture of dynamical evolution" may require:

- More dynamic range in kinematic variables

- Longer time for escaping partons to feel effects of quark-gluon plasma

- Larger samples of charm, bottom, and onium

All these features should be available at the LHC. The table from the talk of Russell Betts[10] shows quantitatively the much higher energy densities, multi- 
Table 1. $\mathrm{Pb}+\mathrm{Pb}$ Collistions at the SPS, RHIC, and LHC

\begin{tabular}{|c|ccc|}
\hline & SPS(17) & RHIC $(200)$ & LHC $(5500)$ \\
\hline$d N_{c h} / d \eta$ & 500 & 700 & $3000-8000$ \\
\hline$\epsilon\left[\mathrm{GeV} / \mathrm{fm}^{3}\right]$ & $\sim 2.5$ & $\sim 3.5-7.5$ & $\sim 15-40$ \\
$\left(t_{0}=1 \mathrm{fm} / \mathrm{c}\right)$ & 1 & 2 & 10 \\
\hline$V_{f}\left[\mathrm{fm}^{3}\right]$ & $\sim 10^{3}$ & $\sim 7 x 10^{3}$ & $\sim 2 x 10^{4}$ \\
& 1 & 7 & 20 \\
\hline$\tau_{Q G P}[\mathrm{fm} / \mathrm{c}]$ & $\leq 1$ & $1.5-4$ & $4-10$ \\
& 1 & 3 & 7 \\
\hline
\end{tabular}

plicities characteristic of more quark-gluon plasma, and the longer times available for the plasma to influence the outgoing states. All these should make the anticipated effects much easier to see at the LHC and to understand.

Finally, I'd like to focus on two personal-favorite physics topics: compossitness and extra dimensions. Signatures for both of these topics may come to be manifest in the same way that high- $p_{t}$ events came to us at the ISR and at Fermilab. Moreover, such signals can appear quite early, with subsets of working detectors and the simplest of analyses.

\section{The Message}

Our keynote speaker, Scott Willenbrock, gave us a similar message to mine. In many ways, physics has never been more exciting.

- We are about to extend the energy frontier by a factor of 7 .

- We have an excellent model of what we have seen already.

- However, we know that our model is incomplete, and we have detailed predictions which soon can be tested definitively.

We are not at the "end of science," but hopefully at the threshold of exciting new science. What will the new science be? I really don't know. However, personally, I expect we will have major surprises. I expect surprises comparable to those when the CERN ISR and Fermilab began. In the face of the new energy frontier;

- be prepared to read out all working detectors;

- be prepared for analysis of early, imperfect data;

- be prepared for discovery;

- be prepared for surprises in signal and in backgrounds;

- and be prepared to think new thoughts!

Good luck! 


\section{A Word of Thanks}

I would like to end with a word of thanks

- to the organizers,

- to the support staff,

- and to the speakers (specially those who responded to my request for advance word on their presentations)

for making this such an informative and invigorating symposium.

\section{References}

1. Production of Hadrons with Large Transverse Momentum at 200 and $300 \mathrm{GeV}$. J.W. Cronin, et al.; Phys. Rev. Lett. 31 (1973) 1426

2. Observation of $\pi^{0}$ Mesons with Large Transverse Momentum in High-Energy Proton Proton Collisions. F.W. Busser, et al.; Phys. Lett. B 46 (1973) 471

3. Vector Meson and Psi Contributions to Single Lepton Spectra. M. Bourquin and J.-M. Gaillard; Phys. Lett. B 59 (1975) 191

4. M. Bishai in these proceedings.

5. L. Bauerdick in these proceedings.

6. N. Brook in these proceedings.

7. J.-M. Qian in these proceedings.

8. See http://lhc-new-homepage.web.cern.ch/lhc-new-homepage/DashBoard/index.asp

9. G. Roland in these proceedings.

10. R. Betts in these proceedings. 